\title{
Biorthogonal wavelets, MRA's and shift-invariant spaces
}

\author{
by
}

\section{Marcin Bownik (Ann Arbor, MI) and Gustavo Garrigós (Madrid)}

\begin{abstract}
We give a characterization of biorthogonal wavelets arising from MRA's of multiplicity $D$ entirely in terms of the dimension function. This improves the previous characterization in [8] removing an unnecessary angle condition. Besides we characterize Riesz wavelets arising from MRA's, and present new proofs based on shift-invariant space theory, generalizing the 1-dimensional results appearing in [17].
\end{abstract}

1. Introduction. Let $\Gamma=A \mathbb{Z}^{n}$ denote a fixed lattice in $\mathbb{R}^{n}$ and $M$ be an expansive matrix preserving the lattice: $M \Gamma \subset \Gamma$. A collection of functions $\Psi=\left\{\psi^{1}, \ldots, \psi^{L}\right\}$ in $L^{2}\left(\mathbb{R}^{n}\right)$ is said to be a (Riesz) wavelet family if the system

$$
\psi_{j, \gamma}^{l}(x)=|\operatorname{det} M|^{j / 2} \psi^{l}\left(M^{j} x-\gamma\right), \quad j \in \mathbb{Z}, \gamma \in \Gamma, l=1, \ldots, L,
$$

is a Riesz basis of $L^{2}\left(\mathbb{R}^{n}\right)$; that is, $\overline{\operatorname{span}}\left\{\psi_{j, \gamma}^{l}\right\}=L^{2}\left(\mathbb{R}^{n}\right)$ and there are constants $a, b>0$ such that

$$
a \sum_{l, j, \gamma}\left|c_{j, \gamma}^{l}\right|^{2} \leq\left\|\sum_{l, j, \gamma} c_{j, \gamma}^{l} \psi_{j, \gamma}^{l}\right\|_{2}^{2} \leq b \sum_{l, j, \gamma}\left|c_{j, \gamma}^{l}\right|^{2}
$$

for every finite sequence of scalars $\left\{c_{j, \gamma}^{l}\right\}$ (see [16, §8.5], [2]). We say that a wavelet family $\Psi$ arises from an MRA (or is associated with an MRA) whenever the family of subspaces

$$
V_{J}=\overline{\operatorname{span}}\left\{\psi_{j, \gamma}^{l} \mid j<J, \gamma \in \Gamma, l=1, \ldots, L\right\}, \quad J \in \mathbb{Z},
$$

forms a multiresolution analysis of $L^{2}\left(\mathbb{R}^{n}\right)$. That is,

(i) $V_{J} \subset V_{J+1}, \overline{\bigcup_{J \in \mathbb{Z}} V_{J}}=L^{2}\left(\mathbb{R}^{n}\right)$;

(ii) $f(x) \in V_{J} \Leftrightarrow f(M x) \in V_{J+1}$;

2000 Mathematics Subject Classification: Primary 42C40.

Key words and phrases: Riesz wavelet, biorthogonal wavelet, multiresolution analysis, shift-invariant space, dimension function.

The first author was partially supported by the NSF grant DMS-0200080. Research of the second author supported by IHP Network "HARP: 2002-2006" (European Commission), and by "Programa Ramón y Cajal" and grant "BHF 2001-0189" (MCyT, Spain). 
(iii) $\exists \varphi^{1}, \ldots, \varphi^{D} \in V_{0}:\left\{\varphi_{\gamma}^{d} \equiv \varphi^{d}(\cdot-\gamma) \mid \gamma \in \Gamma, d=1, \ldots, D\right\}$ is a Riesz basis of $V_{0}$.

As usual, a set of functions $\Phi=\left\{\varphi^{1}, \ldots, \varphi^{D}\right\}$ as in (iii) will be called a scaling family for the MRA, while the integer $D$ denotes the multiplicity of $\left\{V_{J}\right\}_{J \in \mathbb{Z}}$.

In this paper we shall be interested in pairs $(\Psi, \widetilde{\Psi})$ of biorthogonal wavelet families, that is, those wavelet families $\Psi=\left\{\psi^{1}, \ldots, \psi^{L}\right\}, \widetilde{\Psi}=\left\{\widetilde{\psi}^{1}, \ldots, \widetilde{\psi}^{L}\right\}$ satisfying

$$
\left\langle\psi_{j, \gamma}^{l}, \widetilde{\psi}_{j^{\prime}, \gamma^{\prime}}^{l^{\prime}}\right\rangle=\delta_{l, l^{\prime}} \delta_{\gamma, \gamma^{\prime}} \delta_{j, j^{\prime}}, \quad \forall j, j^{\prime} \in \mathbb{Z}, \gamma, \gamma^{\prime} \in \Gamma, l, l^{\prime}=1, \ldots, L,
$$

or equivalently, so that the systems $\left\{\psi_{j, \gamma}^{l}\right\},\left\{\widetilde{\psi}_{j, \gamma}^{l}\right\}$ are dual Riesz bases of $L^{2}\left(\mathbb{R}^{n}\right)$. We say that such a couple $(\Psi, \widetilde{\Psi})$ arises from a pair of biorthogonal $M R A$ 's when the corresponding systems $\left\{V_{J}\right\}_{J \in \mathbb{Z}},\left\{\widetilde{V}_{J}\right\}_{J \in \mathbb{Z}}$ as in (1.1) have respective scaling families $\Phi=\left\{\varphi^{1}, \ldots, \varphi^{D}\right\}, \widetilde{\Phi}=\left\{\widetilde{\varphi}^{1}, \ldots, \widetilde{\varphi}^{D}\right\}$ satisfying

$$
\left\langle\varphi_{\gamma}^{d}, \widetilde{\varphi}_{\gamma^{\prime}}^{d^{\prime}}\right\rangle=\delta_{d, d^{\prime}} \delta_{\gamma, \gamma^{\prime}}, \quad \forall \gamma, \gamma^{\prime} \in \Gamma, d, d^{\prime}=1, \ldots, D .
$$

The concepts of biorthogonal wavelets and MRA's play an important role in applications and have been considerably developed in the literature since the work of Cohen and Daubechies [9, 10].

In a previous paper [8], the second author together with A. Calogero provided a characterization of those pairs of biorthogonal wavelet families $(\Psi, \widetilde{\Psi})$ which arise from biorthogonal MRA's. This result extended to the biorthogonal situation (and higher multiplicity) a well known theorem for dyadic orthonormal wavelets in $L^{2}(\mathbb{R})$, proved independently by G. Gripenberg [14] and X. Wang [21] in the mid-90's (see [16, Theorem 7.3.2] and references therein). In both cases, the characterization relied on a discretization technique introduced by P. Auscher some years before [2], which relates the space $V_{0}$ in (1.1) with the "spaced-valued" function:

$$
\xi \in \mathbb{R}^{n} \mapsto \mathcal{F}(\xi)=\overline{\operatorname{span}}\left\{\left(\widehat{\psi}^{l}\left(M^{* j}(\xi+\gamma)\right)\right)_{\gamma \in \Gamma^{*}}\right\}_{j \geq 1, l=1, \ldots, L} \subseteq \ell^{2}\left(\Gamma^{*}\right),
$$

and similarly $\widetilde{V}_{0}$ with the corresponding $\widetilde{\mathcal{F}}(\xi)$ (with $\Gamma^{*}$ denoting the dual lattice of $\Gamma$ ). Then, the main result in [8] stated that a couple of biorthogonal wavelets $(\Psi, \widetilde{\Psi})$ arises from biorthogonal MRA's if and only if the following two conditions are satisfied:

(I) $\exists D \geq 1: \operatorname{dim} \mathcal{F}(\xi)=\operatorname{dim} \widetilde{\mathcal{F}}(\xi)=D$, a.e. $\xi \in \mathbb{R}^{n} ;$

(II) $\operatorname{ess-inf}_{\xi \in \mathbb{R}^{n}} \operatorname{Angle}(\mathcal{F}(\xi), \widetilde{\mathcal{F}}(\xi))>0$.

As in the theorem of Gripenberg-Wang, the first condition admits an explicit expression in terms of the so-called dimension function: 


$$
D_{\Psi, \widetilde{\Psi}}(\xi):=|\operatorname{det} A|^{-1} \sum_{l=1}^{L} \sum_{j=1}^{\infty} \sum_{\gamma \in \Gamma} \widehat{\widetilde{\psi}}^{l}\left(M^{* j}(\xi+\gamma)\right) \overline{\widehat{\psi}^{l}\left(M^{* j}(\xi+\gamma)\right)},
$$

as a consequence of the identity $\operatorname{dim} \mathcal{F}(\xi)=\operatorname{dim} \widetilde{\mathcal{F}}(\xi)=D_{\Psi, \widetilde{\Psi}}(\xi)$, a.e. $\xi \in$ $\mathbb{R}^{n}$, whenever $\Psi, \widetilde{\Psi}$ are biorthogonal wavelet families (see $[8, \S 2]$ ). The second condition, however, seemed to be a special constraint of the biorthogonal setting, which trivially holds when $\Psi=\widetilde{\Psi}$. In [8] we used the notion of "angle" between two $D$-dimensional spaces $E, F$ (with $E \cap F^{\perp}=\{0\}$ ) given by

$$
\operatorname{Angle}(E, F)=\left\|C_{e, e}^{1 / 2} C_{e, f}^{-1} C_{f, f}^{1 / 2}\right\|^{-1},
$$

where $C_{e, f}=\left(\left\langle e_{i}, f_{j}\right\rangle\right)_{1 \leq i, j \leq D}$ denotes the correlation (or Gramian) matrix associated with two bases $\boldsymbol{e}=\left\{e_{1}, \ldots, e_{D}\right\}, \boldsymbol{f}=\left\{f_{1}, \ldots, f_{D}\right\}$ of $E$ and $F$, respectively. This definition was naturally obtained from the identity $\operatorname{Angle}(E, F)=\left\|S_{E, F}\right\|^{-1}$, where $S_{E, F}$ denotes the projection onto $E$ "parallel" to $F^{\perp}$ (i.e., $\operatorname{ran} S_{E, F}=E$ and $\operatorname{ker} S_{E, F}=F^{\perp}$; see [8, Proposition 4.5]). In [8], however, we were not able to show the independence of conditions (I) and (II), limiting ourselves to find very mild assumptions on the biorthogonal pair $(\Psi, \widetilde{\Psi})$ so that (II) holds (see [8, Cor. 4.17]).

In this paper we show that condition (II) is indeed redundant, and in fact it is just a consequence of the biorthogonality of the wavelet pair $(\Psi, \widetilde{\Psi})$. This was already shown in the 1-dimensional (dyadic) case by H. Kim, R. Kim and J. Lim, in a recent work [17] where an elegant approach to the whole problem is given. This is based on an interpretation of wavelets and MRA's in terms of shift-invariant spaces, as developed in the works of de Boor, DeVore, Ron [3], Aldroubi [1], and others. Our goal in this paper is to combine these ideas with recent results by the first author [4], in order to give a new proof of the main theorem in [8] in which the "angle condition" (II) has been eliminated. As a consequence, we obtain the following new characterization for biorthogonal MRA wavelets, which generalizes to higher multiplicities (and higher dimensions) the results in [17].

Theorem 1.5. Let $\Psi=\left\{\psi^{1}, \ldots, \psi^{L}\right\}, \widetilde{\Psi}=\left\{\widetilde{\psi}^{1}, \ldots, \widetilde{\psi}^{L}\right\}$ be a pair of biorthogonal wavelet families in $L^{2}\left(\mathbb{R}^{n}\right)$. Then, the following statements are equivalent:

(1) $(\Psi, \widetilde{\Psi})$ arise from a pair of biorthogonal MRA's.

(2) $\exists D \geq 1: D_{\Psi, \widetilde{\Psi}}(\xi)=D$, a.e. $\xi \in \mathbb{R}^{n}$.

(3) Either $\Psi$ or $\widetilde{\Psi}$ is associated with an MRA.

(4) Both $\Psi$ and $\widetilde{\Psi}$ are associated with an $M R A$.

We emphasize the crucial role played by the notion of "angle" between shift-invariant spaces, for which we shall use a definition (due to Aldroubi [1]) equivalent to (1.4) in the case of finite-dimensional spaces. 
In addition, we shall combine the ideas in [17] with recent results about generalized MRA's in [5, 18] to obtain as well a characterization for Riesz wavelets arising from an MRA (not necessarily biorthogonal). This will be our Theorem 3.3 below, which we shall furnish with a variety of examples to illustrate the sharpness and independence of the conditions now used.

The paper is organized as follows. In $\S 2$ we present the relevant theory of shift-invariant spaces including an exhaustive study of the concept of "angle". In $\S 3$ we characterize Riesz wavelets arising from MRA's, and leave for $\S 4$ the proof of Theorem 1.5 and some of its corollaries. Finally, a collection of further comments, new examples and open questions is given in $\S 5$.

\section{Shift-invariant spaces}

2.1. General theory. Throughout this paper the Fourier transform is normalized by

$$
\widehat{f}(\xi)=\int_{\mathbb{R}^{n}} f(x) e^{-i\langle x, \xi\rangle} d x, \quad \xi \in \mathbb{R}^{n}, f \in L^{1}\left(\mathbb{R}^{n}\right) .
$$

We denote by $\Gamma^{*}=2 \pi\left(A^{*}\right)^{-1} \mathbb{Z}^{n}$ the dual lattice of $\Gamma$, and by $\mathcal{D}=\left(A^{*}\right)^{-1} \mathbb{T}^{n}$ $=\left(A^{*}\right)^{-1}[0,2 \pi)^{n}$ the fundamental domain of $\Gamma^{*}$. That is, $\{\mathcal{D}+\gamma\}_{\gamma \in \Gamma^{*}}$ forms a partition of $\mathbb{R}^{n}$.

A closed subspace $S$ of $L^{2}\left(\mathbb{R}^{n}\right)$ is said to be $\Gamma$-invariant (or shift-invariant with respect to $\Gamma$ ) if $f(\cdot-\gamma) \in S$ for all $f \in S$ and $\gamma \in \Gamma$. The $\Gamma$-invariant space generated by a family $\Phi \subset L^{2}\left(\mathbb{R}^{n}\right)$ is denoted by

$$
S(\Phi):=\overline{\operatorname{span}}\left\{\varphi_{\gamma}=\varphi(\cdot-\gamma) \mid \gamma \in \Gamma, \varphi \in \Phi\right\} .
$$

It is not difficult to show, using Zorn's lemma, that every $\Gamma$-invariant space $S$ can be written as $S=S(\Phi)$ for a countable family $\Phi \subset L^{2}\left(\mathbb{R}^{n}\right)$. Actually, $\Phi$ can be taken to be orthogonal and so that the system $\left\{\varphi_{\gamma}\right\}_{\gamma \in \Gamma, \varphi \in \Phi}$ is a tight frame of $S$ (see, e.g., [19, Theorem 1.2.10] or [4, Theorem 3.3]).

A main question in the theory of $\Gamma$-invariant spaces is whether these admit an orthonormal basis of the form $\left\{\varphi_{\gamma}\right\}_{\gamma \in \Gamma, \varphi \in \Phi}$ for some $\Phi \subset L^{2}\left(\mathbb{R}^{n}\right)$. In the study of this and other related problems a fundamental role is played by the following isometric isomorphism, which in this paper we shall call the Helson transform:

$$
\mathcal{H}: L^{2}\left(\mathbb{R}^{n}\right) \rightarrow L^{2}\left(\mathcal{D} ; l^{2}\left(\Gamma^{*}\right)\right), \quad f \mapsto \mathcal{H} f(\xi)=(2 \pi)^{-n / 2}(\widehat{f}(\xi+\gamma))_{\gamma \in \Gamma^{*}} .
$$

Following $[3,4,15]$, we define a range function $J$ as any "space-valued" mapping

$$
J: \mathcal{D} \subset \mathbb{R}^{n} \rightarrow\left\{\text { closed subspaces of } \ell^{2}\left(\Gamma^{*}\right)\right\},
$$

and we say that $J$ is measurable when the operator-valued function of the orthogonal projectors $\xi \mapsto P_{J(\xi)}$ is weakly measurable, i.e., $\xi \mapsto\left\langle P_{J(\xi)} \boldsymbol{u}, \boldsymbol{v}\right\rangle$ is measurable for every $\boldsymbol{u}, \boldsymbol{v} \in \ell^{2}\left(\Gamma^{*}\right)$. A fundamental property of the Helson 
transform is that it assigns to every $\Gamma$-invariant space a measurable range function [15, Theorem 8, p. 59].

Proposition 2.1. If $S=S(\Phi)$ for $\Phi \subset L^{2}\left(\mathbb{R}^{n}\right)$ countable, then

$$
J_{S}(\xi):=\overline{\operatorname{span}}\{\mathcal{H} \varphi(\xi) \mid \varphi \in \Phi\}, \quad \xi \in \mathcal{D},
$$

is a measurable range function. Moreover, we can write

$$
S=\left\{f \in L^{2}\left(\mathbb{R}^{n}\right) \mid \mathcal{H} f(\xi) \in J_{S}(\xi) \text { for a.e. } \xi \in \mathcal{D}\right\} .
$$

Observe, in particular, that $J_{S}(\xi)$ is independent (for a.e. $\xi$ ) of the choice of $\Phi$. An elementary proof of Proposition 2.1, which includes as well a converse, can be found in [4, Proposition 1.5]. We shall need two more results.

Proposition 2.2. Suppose $\Phi \subset L^{2}\left(\mathbb{R}^{n}\right)$ is countable. Then the following statements hold:

(1) The system $\left\{\varphi_{\gamma}\right\}_{\gamma \in \Gamma, \varphi \in \Phi}$ is orthonormal in $L^{2}\left(\mathbb{R}^{n}\right)$ if and only if the system $\left\{|\operatorname{det} A|^{-1 / 2} \mathcal{H} \varphi(\xi)\right\}_{\varphi \in \Phi}$ is orthonormal in $\ell^{2}\left(\Gamma^{*}\right)$ for a.e. $\xi \in \mathcal{D}$.

(2) Likewise, $\left\{\varphi_{\gamma}\right\}_{\gamma \in \Gamma, \varphi \in \Phi}$ is a Riesz system in $L^{2}\left(\mathbb{R}^{n}\right)$ if and only if the system $\left\{|\operatorname{det} A|^{-1 / 2} \mathcal{H} \varphi(\xi)\right\}_{\varphi \in \Phi}$ is a Riesz system in $\ell^{2}\left(\Gamma^{*}\right)$ for a.e. $\xi \in \mathcal{D}$.

Proof. A proof in the case when $\Gamma=\mathbb{Z}^{n}$ can be found in [4, Theorem 2.3]. The general situation follows easily by changing variables. Indeed, define the isometry $D_{A} f(x)=|\operatorname{det} A|^{1 / 2} f(A x)$. If $\left\{\varphi_{\gamma}\right\}_{\gamma \in \Gamma, \varphi \in \Phi}$ is an orthonormal system (ONS) then $\left\{D_{A} \varphi_{k}\right\}_{k \in \mathbb{Z}^{n}, \varphi \in \Phi}$ is also an ONS. Now, using the case $\Gamma=\mathbb{Z}^{n}$ this is equivalent to $\left\{|\operatorname{det} A|^{-1 / 2} \mathcal{H} \varphi(\xi)\right\}_{\varphi \in \Phi}$ being an ONS of $\ell^{2}\left(\Gamma^{*}\right)$ for a.e. $\xi \in \mathcal{D}$. The situation for a Riesz system is similar.

Observe from the previous propositions that if $S$ is $\Gamma$-invariant and $\Phi \subset$ $S$ is countable, then $\left\{\varphi_{\gamma}\right\}_{\gamma \in \Gamma, \varphi \in \Phi}$ is an orthonormal basis (or Riesz basis) of $S=S(\Phi)$ if and only if $\left\{|\operatorname{det} A|^{-1 / 2} \mathcal{H} \varphi(\xi)\right\}_{\varphi \in \Phi}$ is a basis for the space $J_{S}(\xi)$, and a.e. $\xi \in \mathcal{D}$. As an application we obtain the following:

Corollary 2.3. Let $\left\{V_{j}\right\}_{j \in \mathbb{Z}}$ be an $M R A$ in $L^{2}\left(\mathbb{R}^{n}\right)$. Then the multiplicity $D$ of $\left\{V_{j}\right\}_{j \in \mathbb{Z}}$ is a constant depending only on the space $V_{0}$, and not on the choice of a scaling family $\Phi$.

The second result characterizes $\Gamma$-invariant spaces with finite multiplicity.

Proposition 2.4. Let $S$ be a $\Gamma$-invariant space. Then $\operatorname{dim} J_{S}(\xi)=D$ $\in \mathbb{Z}_{+}$, a.e. $\xi \in \mathcal{D}$, if and only if there exist $\varphi^{1}, \ldots, \varphi^{D} \in S$ such that $\left\{\varphi_{\gamma}^{d}\right\}_{d=1, \ldots, D, \gamma \in \Gamma}$ is an orthonormal basis $(O N B)$ of $S$.

Proof. If $\operatorname{dim} J_{S}(\xi)=D$, then it is possible to construct measurable $L^{2}\left(\mathcal{D} ; \ell^{2}\left(\Gamma^{*}\right)\right)$-functions $\mathbf{v}^{1}, \ldots, \mathbf{v}^{D}$ such that $\left\{\mathbf{v}^{1}(\xi), \ldots, \mathbf{v}^{D}(\xi)\right\}$ is an ONB of $J_{S}(\xi)$ for a.e. $\xi \in \mathcal{D}$ (see, e.g., [8, Lemma 3.17]). Thus, defining

$$
\widehat{\varphi}^{d}(\xi):=|\operatorname{det} A|^{1 / 2} v_{\gamma}^{d}(\xi-\gamma), \quad \xi \in \mathcal{D}+\gamma, \gamma \in \Gamma^{*}, d=1, \ldots, D,
$$


we have $\mathcal{H} \varphi^{d}=\mathbf{v}^{d}$ and the result follows from the two previous propositions. The converse is also immediate from the two previous propositions.

2.2. The angle between shift-invariant spaces. We begin with the following definition of angle between subspaces of $L^{2}\left(\mathbb{R}^{n}\right)$, taken from the work of Aldroubi $[1,20]$.

Definition 2.5. The angle $R(E, F)$ between two closed subspaces $E, F$ of a Hilbert space $H$ is defined as

$$
R(E, F)=\inf \left\{\frac{\left\|P_{E}(f)\right\|}{\|f\|} \mid f \in F \backslash\{0\}\right\},
$$

where $P_{E}$ denotes the orthogonal projector onto $E$.

We point out that $R(E, F)$ is actually the cosine of the angle $\Theta(E, F)$ between $E$ and $F$ as it is defined in [1], i.e., $\cos \Theta(E, F)=R(E, F)$. Observe also that, even though always $R(E, F)=R\left(F^{\perp}, E^{\perp}\right)$ (see [20, Lemma 1]), in general, we cannot expect that $R(E, F)=R(F, E)$. The following example is illustrative.

Example 2.7. Suppose $Z_{1}, Z_{2}$ are two measurable subsets of $\mathbb{R}^{n}$. Consider the following subspaces of $L^{2}\left(\mathbb{R}^{n}\right)$ :

$$
L_{Z_{1}}^{2}=\left\{f \in L^{2}\left(\mathbb{R}^{n}\right) \mid \operatorname{Supp} \widehat{f} \subset Z_{1}\right\}, \quad L_{Z_{2}}^{2}=\left\{f \in L^{2}\left(\mathbb{R}^{n}\right) \mid \operatorname{Supp} \widehat{f} \subset Z_{2}\right\},
$$

which are actually invariant with respect to all translations in $\mathbb{R}^{n}$. Now, if $Z_{2} \subset Z_{1}$ (modulo null sets) then $R\left(L_{Z_{1}}^{2}, L_{Z_{2}}^{2}\right)=1$. Otherwise $R\left(L_{Z_{1}}^{2}, L_{Z_{2}}^{2}\right)=0$.

The next general result gives an explicit expression for the angle between spaces with the same finite dimension, showing that in this case $R(E, F)=$ $R(F, E)=\operatorname{Angle}(E, F)$, as defined in (1.4).

Proposition 2.8. Let $E, F$ be vector subspaces of a Hilbert space $H$ with $\operatorname{dim} E=\operatorname{dim} F=d$, and $\boldsymbol{e}=\left\{e_{1}, \ldots, e_{d}\right\}, \boldsymbol{f}=\left\{f_{1}, \ldots, f_{d}\right\}$ be their respective orthonormal bases. If $E \cap F^{\perp}=\{0\}$, then

$$
R(E, F)=\left\|C_{e, f}^{-1}\right\|^{-1},
$$

where $C_{e, f}=\left(\left\langle e_{i}, f_{j}\right\rangle\right)_{1 \leq i, j \leq d}$ is the correlation matrix of $\boldsymbol{e}$ and $\boldsymbol{f}$.

Proof. Take any $y=\sum_{j=1}^{d} y_{j} f_{j} \in F$, where $y_{j}=\left\langle y, f_{j}\right\rangle$, and observe that, from the formula for the projector $P_{E} y=\sum_{i=1}^{d}\left\langle y, e_{i}\right\rangle e_{i}$, we have

$$
\left\|P_{E} y\right\|^{2}=\left\|\sum_{j=1}^{d} y_{j} \sum_{i=1}^{d}\left\langle f_{j}, e_{i}\right\rangle e_{i}\right\|^{2}=\sum_{i=1}^{d}\left|\sum_{j=1}^{d} y_{j}\left\langle f_{j}, e_{i}\right\rangle\right|^{2}=\left|C_{\boldsymbol{e}, \boldsymbol{f}} \mathbf{Y}\right|^{2},
$$

where $\mathbf{Y}$ denotes the column matrix with entries $y_{1}, \ldots, y_{d}$. As $E \cap F^{\perp}=\{0\}$, 
the matrix $C_{e, f}$ is invertible (see, e.g., [8, Lemma 4.3]) and therefore

$$
\begin{aligned}
\inf _{y \in F \backslash\{0\}} \frac{\left\|P_{E} y\right\|}{\|y\|} & =\inf _{\left(y_{1}, \ldots, y_{d}\right) \in \mathbb{C}^{d} \backslash\{0\}} \frac{\left|C_{e, f} \mathbf{Y}\right|}{|\mathbf{Y}|} \\
& =\left[\sup _{\left(z_{1}, \ldots, z_{d}\right) \in \mathbb{C}^{d} \backslash\{0\}} \frac{\left|C_{e, f}^{-1} \mathbf{Z}\right|}{|\mathbf{Z}|}\right]^{-1}=\left\|C_{e, f}^{-1}\right\|^{-1},
\end{aligned}
$$

where $\mathbf{Z}$ is the column matrix with entries $z_{1}, \ldots, z_{d}$.

Corollary 2.9. Let $E, F$ be subspaces of a Hilbert space $H$ with the same finite dimension, or the same finite co-dimension. Then $R(E, F)=$ $R(F, E)$.

Our next results concern the angle between $\Gamma$-invariant spaces.

Proposition 2.10. Suppose $S_{1}, S_{2}$ are two $\Gamma$-invariant spaces of $L^{2}\left(\mathbb{R}^{n}\right)$. Then

$$
R\left(S_{1}, S_{2}\right)=\underset{\xi \in \mathcal{D}}{\operatorname{ess-inf}} R\left(J_{S_{1}}(\xi), J_{S_{2}}(\xi)\right)
$$

Proof. Let $P_{S_{1}}$ be the orthogonal projector of $L^{2}\left(\mathbb{R}^{n}\right)$ onto $S_{1}$, and denote by $P_{1}(\xi)=P_{J_{S_{1}}(\xi)}$ the corresponding orthogonal projector from $\ell^{2}\left(\Gamma^{*}\right)$ onto $J_{S_{1}}(\xi)$. Then, for every $f \in L^{2}\left(\mathbb{R}^{n}\right)$ the Helson transform gives the equality

$$
\mathcal{H}\left(P_{S_{1}} f\right)(\xi)=P_{1}(\xi)(\mathcal{H} f(\xi)), \quad \text { a.e. } \xi \in \mathcal{D}
$$

(see, e.g., [4, Lemma 1.4]). Further, restricting the above to $f \in S_{2}$ we see that

$$
\begin{aligned}
\mathcal{H}\left(\left.P_{S_{1}}\right|_{S_{2}} f\right)(\xi) & =\mathcal{H}\left(P_{S_{1}} P_{S_{2}} f\right)(\xi) \\
& =P_{1}(\xi) P_{2}(\xi)(\mathcal{H} f(\xi))=\left.P_{1}(\xi)\right|_{J_{S_{2}}(\xi)}(\mathcal{H} f(\xi)),
\end{aligned}
$$

for a.e. $\xi \in \mathcal{D}$. Thus, in the notation of $[4, \S 4],\left.P_{1}(\xi)\right|_{J_{S_{2}}(\xi)}$ is the range operator corresponding to $\left.P_{S_{1}}\right|_{S_{2}}$ in the $\Gamma$-invariant space $S_{2}$. Now we can use [4, Theorem 4.6] which states:

$$
\begin{aligned}
\inf \left\{\frac{\left\|P_{S_{1}}(f)\right\|}{\|f\|} \mid f \in S_{2} \backslash\{0\}\right\} & \\
& =\underset{\xi \in \mathcal{D}}{\operatorname{ess-inf}} \inf \left\{\frac{\left\|P_{1}(\xi) v\right\|}{\|v\|} \mid v \in J_{S_{2}}(\xi) \backslash\{0\}\right\},
\end{aligned}
$$

establishing (2.11).

As an immediate consequence of Proposition 2.10 and Corollary 2.9 we have the following.

Corollary 2.12. Let $S_{1}, S_{2}$ be $\Gamma$-invariant spaces of $L^{2}\left(\mathbb{R}^{n}\right)$ satisfying $\operatorname{dim} J_{S_{1}}(\xi)=\operatorname{dim} J_{S_{2}}(\xi)<\infty \quad$ or $\quad \operatorname{dim} J_{S_{1}}(\xi)^{\perp}=\operatorname{dim} J_{S_{2}}(\xi)^{\perp}<\infty$ for a.e. $\xi \in \mathcal{D}$. Then $R\left(S_{1}, S_{2}\right)=R\left(S_{2}, S_{1}\right)$. 
We conclude with the following result, giving necessary and sufficient conditions for the existence of biorthogonal Riesz bases for two $\Gamma$-invariant spaces.

Proposition 2.13. Let $D \in \mathbb{Z}^{+}$and $S_{1}, S_{2}$ be $\Gamma$-invariant spaces of $L^{2}\left(\mathbb{R}^{n}\right)$ satisfying

$$
\operatorname{dim} J_{S_{1}}(\xi)=\operatorname{dim} J_{S_{2}}(\xi)=D \quad \text { for a.e. } \xi \in \mathcal{D} .
$$

If $R\left(S_{1}, S_{2}\right)>0$, then for any $\varphi^{1}, \ldots, \varphi^{D} \in S_{1}$ so that $\left\{\varphi_{\gamma}^{d}\right\}_{d=1, \ldots, D, \gamma \in \Gamma}$ is a Riesz basis of $S_{1}$ (with constants $a, b$ ), there exist $\widetilde{\varphi}^{1}, \ldots, \widetilde{\varphi}^{D} \in S_{2}$ so that $\left\{\widetilde{\varphi}_{\gamma}^{d}\right\}_{d=1, \ldots, D, \gamma \in \Gamma}$ is a Riesz basis of $S_{2}$ (with constants $b^{-1}, R\left(S_{2}, S_{1}\right)^{-1 / 2} a^{-1}$ ) and satisfying the biorthogonality relation

$$
\left\langle\varphi_{\gamma}^{d}, \widetilde{\varphi}_{\gamma^{\prime}}^{d^{\prime}}\right\rangle=\delta_{d, d^{\prime}} \delta_{\gamma, \gamma^{\prime}}, \quad d, d^{\prime}=1, \ldots, D, \gamma, \gamma^{\prime} \in \Gamma .
$$

Conversely, if $\left\{\varphi_{\gamma}^{d}\right\}_{d=1, \ldots, D, \gamma \in \Gamma},\left\{\widetilde{\varphi}_{\gamma}^{d}\right\}_{d=1, \ldots, D, \gamma \in \Gamma}$ are biorthogonal Riesz bases of $S_{1}$ and $S_{2}$, then (2.14) holds and $R\left(S_{1}, S_{2}\right)=R\left(S_{2}, S_{1}\right)>0$.

Proof. Since $R\left(S_{2}, S_{1}\right)>0$,

$$
R\left(S_{2}, S_{1}\right)\|f\| \leq\left\|P_{S_{2}} f\right\| \leq\|f\|, \quad \forall f \in S_{1} .
$$

Thus, if $\left\{\varphi_{\gamma}^{d}\right\}_{d, \gamma}$ is a Riesz basis for $S_{1}$ (with constants $a, b$ ), so is the system $\left\{P_{S_{2}}\left(\varphi_{\gamma}^{d}\right)=\left(P_{S_{2}} \varphi^{d}\right)_{\gamma}\right\}_{d, \gamma}$ for $S_{2}$ (with constants $\left.R\left(S_{2}, S_{1}\right)^{1 / 2} a, b\right)$. In this last claim it should be observed that $\overline{\operatorname{span}}\left\{P_{S_{2}}\left(\varphi_{\gamma}^{d}\right)\right\}_{d, \gamma}=S_{2}$ because of $R\left(S_{1}, S_{2}\right)>0$ (alternatively, one can use (2.14)). Now, we invoke [4, Theorem 5.2 (and the subsequent Remark)], which guarantees the existence of a dual Riesz basis for $\left\{P_{S_{2}}\left(\varphi_{\gamma}^{d}\right)\right\}_{d, \gamma}$ in $S_{2}$ of the form $\left\{\widetilde{\varphi}_{\gamma}^{d}\right\}_{d, \gamma}$ (with constants $\left.b^{-1}, R\left(S_{2}, S_{1}\right)^{-1 / 2} a^{-1}\right)$. Thus,

$$
\left\langle\varphi_{\gamma}^{d}, \widetilde{\varphi}_{\gamma^{\prime}}^{d^{\prime}}\right\rangle=\left\langle\varphi_{\gamma}^{d}, P_{S_{2}}\left(\widetilde{\varphi}_{\gamma^{\prime}}^{d^{\prime}}\right)\right\rangle=\left\langle P_{S_{2}}\left(\varphi_{\gamma}^{d}\right), \widetilde{\varphi}_{\gamma^{\prime}}^{d^{\prime}}\right\rangle=\delta_{d, d^{\prime}} \delta_{\gamma, \gamma^{\prime}}
$$

establishing the first part of the proposition.

Conversely, suppose $\left\{\varphi_{\gamma}^{d}\right\}_{d, \gamma},\left\{\widetilde{\varphi}_{\gamma}^{d}\right\}_{d, \gamma}$ are Riesz bases (with constants $a, b$ and $\widetilde{a}, \widetilde{b})$ of $S_{1}, S_{2}$, resp., which are biorthogonal, i.e., (2.15) holds. By the second statement of Proposition 2.2, $\left\{|\operatorname{det} A|^{-1 / 2} \mathcal{H} \varphi^{d}(\xi)\right\}_{d=1}^{D}$ is a Riesz basis for $J_{S_{1}}(\xi)$, for a.e. $\xi \in \mathcal{D}$, and hence $\operatorname{dim} J_{S_{1}}(\xi)=D$ for a.e. $\xi \in \mathcal{D}$. Analogously, $\operatorname{dim} J_{S_{2}}(\xi)=D$ for a.e. $\xi \in \mathcal{D}$, which shows (2.14). It remains to show that $R\left(S_{1}, S_{2}\right)=R\left(S_{2}, S_{1}\right)>0$.

By [4, Theorem 5.2 (and the subsequent Remark)] the dual Riesz basis of $\left\{\varphi_{\gamma}^{d}\right\}_{d, \gamma}$ in $S_{1}$ is again of the form $\left\{\varphi_{\gamma}^{\sharp d}\right\}_{d, \gamma}$ for some $\left\{\varphi^{\sharp 1}, \ldots, \varphi^{\sharp D}\right\} \subset S_{1}$. Therefore, the orthogonal projection $P_{S_{1}}$ onto $S_{1}$ can be given as

$$
P_{S_{1}} f=\sum_{d=1}^{D} \sum_{\gamma \in \Gamma}\left\langle f, \varphi_{\gamma}^{\sharp d}\right\rangle \varphi_{\gamma}^{d}=\sum_{d=1}^{D} \sum_{\gamma \in \Gamma}\left\langle f, \varphi_{\gamma}^{d}\right\rangle \varphi_{\gamma}^{\sharp d} \quad \text { for all } f \in L^{2}\left(\mathbb{R}^{n}\right) .
$$


Observe in particular that $P_{S_{1}} \widetilde{\varphi}_{\gamma}^{d}=\varphi_{\gamma}^{\sharp d}$ for all $\gamma, d$. Pick now a function $f \in S_{2} \backslash\{0\}$, and expand it as

$$
f=\sum_{d=1}^{D} \sum_{\gamma \in \Gamma} c_{\gamma}^{d} \widetilde{\varphi}_{\gamma}^{d}, \quad \text { where } \quad \widetilde{a} \sum_{d=1}^{D} \sum_{\gamma \in \Gamma}\left|c_{\gamma}^{d}\right|^{2} \leq\|f\|^{2} \leq \widetilde{b} \sum_{d=1}^{D} \sum_{\gamma \in \Gamma}\left|c_{\gamma}^{d}\right|^{2} .
$$

Then $P_{S_{1}} f=\sum_{d=1}^{D} \sum_{\gamma \in \Gamma} c_{\gamma}^{d} \varphi_{\gamma}^{\sharp d}$, and

$$
\frac{\left\|P_{S_{1}} f\right\|^{2}}{\|f\|^{2}} \geq \frac{b^{-1} \sum_{d, \gamma}\left|c_{\gamma}^{d}\right|^{2}}{\widetilde{b} \sum_{d, \gamma}\left|c_{\gamma}^{d}\right|^{2}}=\frac{1}{\overrightarrow{b b}}
$$

since $\left\{\varphi_{\gamma}^{\sharp d}\right\}$ is a Riesz basis with constants $b^{-1}, a^{-1}$. This shows $R\left(S_{1}, S_{2}\right) \geq$ $(\widetilde{b})^{-1 / 2}$ and completes the proof of Proposition 2.13.

REMARK 2.16. The previous proposition holds as well (and with the same proof) when we let $D=\infty$ in (2.14). In this case, the condition $R\left(S_{1}, S_{2}\right)>0$ in the first statement must be replaced by $R\left(S_{1}, S_{2}\right) R\left(S_{2}, S_{1}\right)>0$.

As an application to MRA theory we have the following characterization.

Corollary 2.17. Let $\left\{V_{j}\right\}_{j \in \mathbb{Z}},\left\{\widetilde{V}_{j}\right\}_{j \in \mathbb{Z}}$ be two multiresolution analyses in $L^{2}\left(\mathbb{R}^{n}\right)$ with the same multiplicity. Then $\left\{V_{j}, \widetilde{V}_{j}\right\}_{j \in \mathbb{Z}}$ are biorthogonal if and only if $R\left(V_{0}, \widetilde{V}_{0}\right)>0$.

The previous corollary tells us that for a given multiresolution analysis $\left\{V_{j}\right\}_{j \in \mathbb{Z}}$, there may be many other MRA's $\left\{\widetilde{V}_{j}\right\}_{j \in \mathbb{Z}}$ so that $\left\{V_{j}, \widetilde{V}_{j}\right\}_{j \in \mathbb{Z}}$ are biorthogonal. In particular, we can produce a large collection of biorthogonal wavelets from a fixed scaling function $\varphi$, by just choosing appropriate biorthogonal scaling functions $\widetilde{\varphi}$. This contrasts with the fact that a Riesz wavelet $\psi$ can have at most one partner $\widetilde{\psi}$ so that $(\psi, \widetilde{\psi})$ are biorthogonal wavelets. The following example (in the 1-dimensional dyadic case) is quite illustrative.

EXAMPLE 2.18: The Haar and Shannon MRA's in $L^{2}(\mathbb{R})$. Following the notation in [16, Ch. 2], we define the Haar $M R A$ from $V_{0}=S(\varphi)$, where $\varphi=$ $\chi_{[-1,0]}$ is the Haar scaling function. Analogously, we consider the Shannon $M R A$, letting $V_{0}^{\sharp}=S\left(\varphi^{\sharp}\right)$ with $\widehat{\varphi}^{\sharp}=\chi_{[-\pi, \pi]}$. It is well known that these two MRA's lead to the Haar and Shannon (orthonormal) wavelets, respectively. Observe now that

$$
\begin{aligned}
R\left(V_{0}, V_{0}^{\sharp}\right) & =\operatorname{ess-inf}_{\xi \in[-\pi, \pi)}\left|\sum_{k \in \mathbb{Z}} \widehat{\varphi}^{\sharp}(\xi+2 k \pi) \overline{\widehat{\varphi}(\xi+2 k \pi)}\right| \\
& =\inf _{\xi \in[-\pi, \pi)} \frac{\sin \xi / 2}{\xi / 2}=\frac{2}{\pi}>0,
\end{aligned}
$$

and therefore, $\left\{V_{j}, V_{j}^{\sharp}\right\}$ are biorthogonal MRA's. Thus, we can also construct 
a pair of biorthogonal wavelets following the classical Cohen-Daubechies procedure $[9,10]$. First let $\widehat{\widetilde{\varphi}}=\widehat{\varphi}^{\sharp} / \overline{\widehat{\varphi}}$, so that $\{\varphi, \widetilde{\varphi}\}$ are biorthogonal scaling functions. Now, consider the low-pass filters:

$$
m_{0}(\xi)=\frac{1+e^{i \xi}}{2} \text { and } \quad \widetilde{m}_{0}(\xi)=\frac{\chi_{[-\pi / 2, \pi / 2]+2 \pi \mathbb{Z}}(\xi)}{\overline{m_{0}(\xi)}},
$$

so that the following scaling equations hold:

$$
\widehat{\varphi}(2 \xi)=m_{0}(\xi) \widehat{\varphi}(\xi) \quad \text { and } \quad \widehat{\widetilde{\varphi}}(2 \xi)=\widetilde{m}_{0}(\xi) \widehat{\widetilde{\varphi}}(\xi), \quad \text { a.e. } \xi \in \mathbb{R} .
$$

Next, we define the high-pass filters:

$$
m_{1}(\xi)=e^{i \xi} \overline{m_{0}(\xi+\pi)} \quad \text { and } \quad \widetilde{m}_{1}(\xi)=e^{i \xi} \overline{m_{0}(\xi+\pi)} .
$$

Then the candidates for biorthogonal wavelets are:

$$
\begin{aligned}
\widehat{\psi}(\xi) & =m_{1}(\xi / 2) \widehat{\varphi}(\xi / 2) \\
& =e^{i \xi / 2} \frac{\widehat{\varphi}(\xi / 2)}{m_{0}(\xi / 2+\pi)} \chi_{[\pi, 3 \pi]+4 \pi \mathbb{Z}}(\xi)=i e^{i \xi / 2} \frac{4}{\xi} \chi_{[\pi, 3 \pi]+4 \pi \mathbb{Z}}(\xi)
\end{aligned}
$$

and

$$
\begin{aligned}
\widehat{\widetilde{\psi}}(\xi) & =\widetilde{m}_{1}(\xi / 2) \widehat{\widetilde{\varphi}}(\xi / 2) \\
& =e^{i \xi / 2} \overline{\left(\frac{m_{0}(\xi / 2+\pi)}{\widehat{\varphi}(\xi / 2)}\right)} \chi_{[-2 \pi, 2 \pi]}(\xi)=i e^{i \xi / 2} \frac{\xi}{4} \chi_{[-2 \pi, 2 \pi]}(\xi) .
\end{aligned}
$$

We observe that, although with this definition the systems $\left\{\psi_{j, k}\right\}_{j, k \in \mathbb{Z}}$, $\left\{\widetilde{\psi}_{j, k}\right\}_{j, k \in \mathbb{Z}}$ are always biorthogonal, one has to check separately that they satisfy the Bessel property, and hence, constitute a pair of dual Riesz bases in $L^{2}(\mathbb{R})$. Now, following [12, p. 268], the Bessel property for a sequence $\left\{\varrho_{j, k}\right\}_{j, k \in \mathbb{Z}}$ in $L^{2}(\mathbb{R})$ holds provided the function $\widehat{\varrho}$ satisfies the two estimates

$$
\sum_{k \in \mathbb{Z}}|\widehat{\varrho}(\xi+2 \pi k)|^{2(1-\varepsilon)} \leq C \quad \text { and } \quad \sum_{j \in \mathbb{Z}}\left|\widehat{\varrho}\left(2^{j} \xi\right)\right|^{2 \varepsilon} \leq C, \quad \text { a.e. } \xi \in \mathbb{R},
$$

for some constants $\varepsilon, C>0$. This condition is easily verified for $\varrho=\psi, \widetilde{\psi}$, when $0<\varepsilon<1 / 2$, and thus $(\psi, \widetilde{\psi})$ are biorthogonal wavelets.

Finally, it is very easy to construct examples of non-biorthogonal MRA's.

EXAMPLE 2.20: Non-biorthogonal MRA's. Consider the following perturbations of the Shannon scaling function: $\widehat{\varphi}^{(a)}=\chi_{[-a, 2 \pi-a]}, 0<a<2 \pi$. Then the basic MRA spaces take the form $V_{0}^{(a)}=S\left(\varphi^{(a)}\right)=L_{[-a, 2 \pi-a]}^{2}$, in the notation of Example 2.7. Thus

$$
R\left(V_{0}^{(a)}, V_{0}^{\left(a^{\prime}\right)}\right)=0, \quad \forall a \neq a^{\prime},
$$

and the pair of MRA's $\left\{V_{j}^{(a)}, V_{j}^{\left(a^{\prime}\right)}\right\}_{j \in \mathbb{Z}}$ cannot be biorthogonal.

It is also possible to obtain an MRA which is not biorthogonal to the Haar MRA. For this we can consider a scaling function $\widehat{\varphi}^{K}=\chi_{K}$, where $K$ 
is a suitable "scaling set" having a point of density at $\xi=2 \pi$. In this way, $V_{0}^{K}=S\left(\varphi^{K}\right)=L_{K}^{2}$ and

$$
R\left(V_{0}, V_{0}^{K}\right)=\inf _{\xi \in K} \frac{\sin \xi / 2}{\xi / 2}=0 .
$$

Examples of such pathological scaling sets $K$ are presented in [7, Prop. C].

3. Riesz wavelets. Let now $\Psi=\left\{\psi^{1}, \ldots, \psi^{L}\right\}$ be a Riesz wavelet family and $\left\{V_{J}\right\}_{J \in \mathbb{Z}}$ the collection of subspaces defined in (1.1). Recall from (1.2) the definition of the spaces $\mathcal{F}(\xi)=\overline{\operatorname{span}}\left\{\mathcal{H} \psi_{j, 0}^{l}(\xi)\right\}_{l=1, \ldots, L, j<0}$. Following $[17$, $\S 2]$ we observe that

$$
S\left(V_{0}\right)=S\left(\left\{\psi_{j, 0}^{l}\right\}_{l=1, \ldots, L, j<0}\right)
$$

Indeed, the inclusion " $\supseteq$ " is obvious from $V_{0} \supseteq\left\{\psi_{j, 0}^{l}\right\}_{l=1, \ldots, L, j<0}$. Conversely,

$$
\begin{aligned}
V_{0} & =\overline{\operatorname{span}}\left\{\psi_{j, \gamma}^{l}\right\}_{l=1, \ldots, L, j<0, \gamma \in \Gamma} \\
& \subseteq \overline{\operatorname{span}}\left\{\psi^{l}\left(M^{* j}(\cdot+\gamma)\right)\right\}_{l=1, \ldots, L, j<0, \gamma \in \Gamma}=S\left(\left\{\psi_{j, 0}^{l}\right\}_{l=1, \ldots, L, j<0}\right),
\end{aligned}
$$

and therefore " $\subseteq$ " holds in (3.1). In particular, we conclude from Proposition 2.1 that

$$
J_{S\left(V_{0}\right)}(\xi)=\mathcal{F}(\xi), \quad \text { a.e. } \xi \in \mathcal{D} .
$$

The following result appears to be new in the literature and generalizes $[17$, Theorem 2.6].

THEOREM 3.3. Let $\Psi=\left\{\psi^{1}, \ldots, \psi^{L}\right\}$ be a Riesz wavelet family. Then $\Psi$ is associated with an MRA if and only if $V_{0}$ given by (1.1) is $\Gamma$-invariant and there exists an integer $D \geq 1$ such that $\operatorname{dim} \mathcal{F}(\xi)=D$, a.e. $\xi \in \mathcal{D}$. In this case, $L=D(|\operatorname{det} M|-1)$ and $\mathcal{F}(\xi)=J_{V_{0}}(\xi)$, a.e. $\xi \in \mathcal{D}$.

Proof. The necessary condition is easy. If $\Psi$ is associated with an MRA, then there exist $\varphi^{1}, \ldots, \varphi^{D} \in V_{0}$ such that $\left\{\varphi_{\gamma}^{d}\right\}_{d, \gamma}$ is a Riesz basis of $V_{0}$. Hence, $V_{0}$ is $\Gamma$-invariant and $S\left(V_{0}\right)=V_{0}$. Moreover, from Propositions 2.1 and 2.2, $\left\{\mathcal{H} \varphi^{d}(\xi)\right\}_{d=1}^{D}$ is also a Riesz basis of $J_{V_{0}}(\xi)=\mathcal{F}(\xi)$, a.e. $\xi \in \mathcal{D}$, implying $\operatorname{dim} \mathcal{F}(\xi)=D$, a.e. $\xi \in \mathcal{D}$.

Conversely, if $V_{0}$ is $\Gamma$-invariant and $\operatorname{dim} J_{V_{0}}(\xi)=\operatorname{dim} \mathcal{F}(\xi)=D$, a.e. $\xi \in \mathcal{D}$, then by Proposition 2.2, $V_{0}$ has an ONB of the form $\left\{\varphi_{\gamma}^{d}\right\}_{d, \gamma}$, and hence $\left\{V_{J}\right\}_{J \in \mathbb{Z}}$ is an MRA. To show that $L=D(|\operatorname{det} M|-1)$ we quote the following result from [18].

ThEOREM 3.4. Let $\Psi=\left\{\psi^{1}, \ldots, \psi^{L}\right\}$ be a Riesz wavelet family. Then the following statements are equivalent:

(1) There exists $\widetilde{\Psi}=\left\{\widetilde{\psi}^{1}, \ldots, \widetilde{\psi}^{L}\right\} \subset L^{2}\left(\mathbb{R}^{n}\right)$ such that $\left\{\widetilde{\psi}_{j, \gamma}^{l}\right\}_{l, j, \gamma}$ is a dual Riesz basis of $\left\{\psi_{j, \gamma}^{l}\right\}_{l, j, \gamma}$.

(2) $V_{0}$ is $\Gamma$-invariant. 
(3) There exists $\Psi^{\sharp}=\left\{\psi_{1}^{\sharp}, \ldots, \psi_{L}^{\sharp}\right\} \subset L^{2}\left(\mathbb{R}^{n}\right)$ such that $\left\{\psi_{l ; j, \gamma}^{\sharp}\right\}_{l, j, \gamma}$ is an $O N B$ of $L^{2}\left(\mathbb{R}^{n}\right)$ and

$$
V_{J}=V_{J}^{\sharp}:=\overline{\operatorname{span}}\left\{\psi_{l ; j, \gamma}^{\sharp} \mid j<J, \gamma \in \Gamma, l=1, \ldots, L\right\}, \quad \forall J \in \mathbb{Z} .
$$

Going back to Theorem 3.3, since $V_{0}$ is $\Gamma$-invariant, it must equal $V_{0}^{\sharp}$, and therefore,

$$
D=\operatorname{dim} \mathcal{F}(\xi)=\operatorname{dim} J_{V_{0}}(\xi)=\operatorname{dim} J_{V_{0}^{\sharp}}(\xi)=\operatorname{dim} \mathcal{F}^{\sharp}(\xi), \quad \text { a.e. } \xi \in \mathcal{D} .
$$

Thus, using the formula for the dimension function of an orthonormal wavelet family (see, e.g., $[8, \S 2]$ ), we must have

$$
\begin{aligned}
D & =\operatorname{dim} \mathcal{F}^{\sharp}(\xi)=D_{\Psi^{\sharp}}(\xi) \\
& =|\operatorname{det} A|^{-1} \sum_{l=1}^{L} \sum_{j=1}^{\infty} \sum_{\gamma \in \Gamma}\left|\widehat{\psi}_{l}^{\sharp}\left(M^{* j}(\xi+\gamma)\right)\right|^{2}, \quad \text { a.e. } \xi \in \mathcal{D} .
\end{aligned}
$$

Integrating over $\mathcal{D}$ and deperiodizing the integral on the right we obtain

$$
\begin{aligned}
D & =\sum_{l=1}^{L} \sum_{j=1}^{\infty} \sum_{\gamma \in \Gamma} \int_{\mathcal{D}}\left|\widehat{\psi}_{l}^{\sharp}\left(M^{* j}(\xi+\gamma)\right)\right|^{2} d \xi \\
& =\sum_{l=1}^{L} \sum_{j=1}^{\infty}|\operatorname{det} M|^{-j}\left\|\psi_{l}^{\sharp}\right\|_{2}^{2}=\frac{L}{|\operatorname{det} M|-1} . \cdot
\end{aligned}
$$

In the particular case $L=|\operatorname{det} M|-1$, Theorem 3.3 admits a more explicit statement where $\operatorname{dim} \mathcal{F}(\xi)=D$ is replaced by a simpler verifiable condition in terms of $\Psi$.

Theorem 3.5. Suppose $L=|\operatorname{det} M|-1$. If $\Psi=\left\{\psi^{1}, \ldots, \psi^{L}\right\}$ is a Riesz wavelet family, then $\Psi$ is associated with an MRA if and only if $V_{0}$ is $\Gamma$ invariant and

$$
\sum_{l=1}^{L} \sum_{j=1}^{\infty} \sum_{\gamma \in \Gamma}\left|\widehat{\psi}^{l}\left(M^{* j}(\xi+\gamma)\right)\right|^{2}>0, \quad \text { a.e. } \xi \in \mathcal{D} .
$$

In this case, the MRA $\left\{V_{J}\right\}_{J \in \mathbb{Z}}$ has multiplicity 1.

Proof. The direct implication is easy. If $\Psi$ arises from an MRA, we know from Theorem 3.3 that $\operatorname{dim} \mathcal{F}(\xi)=D$, a.e. $\xi \in \mathcal{D}$, for some $D \geq 1$. By the definition of $\mathcal{F}(\xi)$ (see (1.2)) this implies that some vector

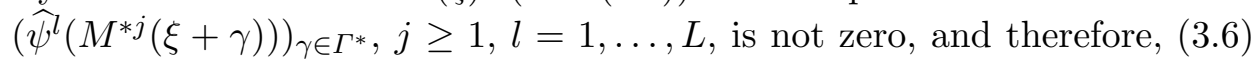
must hold. Conversely, if (3.6) holds, then $\operatorname{dim} \mathcal{F}(\xi) \geq 1$, a.e. $\xi \in \mathcal{D}$. Moreover, if $V_{0}$ is $\Gamma$-invariant we can use Theorem 3.4 which guarantees the existence of a Riesz wavelet family $\widetilde{\Psi}=\left\{\widetilde{\psi}^{1}, \ldots, \widetilde{\psi}^{L}\right\}$ so that $(\Psi, \widetilde{\Psi})$ are biorthogonal wavelet families. Proceeding as in the last step of Theorem 3.3 
we see that

$$
\int_{\mathcal{D}} D_{\Psi, \widetilde{\Psi}}(\xi) \frac{d \xi}{|\mathcal{D}|}=\sum_{l=1}^{L} \frac{\left\langle\psi^{l}, \widetilde{\psi}^{l}\right\rangle}{|\operatorname{det} M|-1}=1 .
$$

On the other hand, as we mentioned in $\S 1$ (see also $(4.1)), D_{\Psi, \widetilde{\Psi}}(\xi)=$ $\operatorname{dim} \mathcal{F}(\xi) \geq 1$, a.e. $\xi \in \mathcal{D}$, and therefore necessarily $\operatorname{dim} \mathcal{F}(\xi)=D_{\Psi, \widetilde{\Psi}}(\xi) \equiv 1$.

REMARK 3.7. As pointed out in [17], several examples in the 1-dimensional dyadic case show that the two conditions in Theorem 3.5 are independent of each other. More examples in the higher-dimensional situation are presented below in $\S 5$.

4. Biorthogonal Riesz wavelets. In this section we shall prove our main result: Theorem 1.5 . We assume throughout this section that $(\Psi, \widetilde{\Psi})$ is a pair of biorthogonal wavelet families in $L^{2}\left(\mathbb{R}^{n}\right)$. Recall from $[8, \S 2]$ that in this case the dimension of the spaces $\mathcal{F}(\xi), \widetilde{\mathcal{F}}(\xi)$ can be explicitly computed:

$$
\operatorname{dim} \mathcal{F}(\xi)=\operatorname{dim} \widetilde{\mathcal{F}}(\xi)=D_{\Psi, \widetilde{\Psi}}(\xi), \quad \text { a.e. } \xi \in \mathbb{R}^{n}
$$

where $D_{\Psi, \widetilde{\Psi}}(\xi)$ is the dimension function defined in (1.3).

Let $Z=\overline{\operatorname{span}}\left\{\psi_{j, \gamma}^{l} \mid j \geq 0, \gamma \in \Gamma, l=1, \ldots, L\right\}$. Then, since $\left\{\psi_{j, \gamma}^{l}\right\}_{l, j, \gamma}$ and $\left\{\widetilde{\psi}_{j, \gamma}^{l}\right\}_{l, j, \gamma}$ are dual Riesz bases for $L^{2}\left(\mathbb{R}^{n}\right)$ it follows that

$$
L^{2}\left(\mathbb{R}^{n}\right)=V_{0} \oplus Z=\widetilde{V}_{0} \stackrel{\perp}{\oplus} Z,
$$

where $\widetilde{V}_{0}=\overline{\operatorname{span}}\left\{\widetilde{\psi}_{j, \gamma}^{l}\right\}_{l=1, \ldots, L, j<0, \gamma \in \Gamma}$, the first sum is direct and the second orthogonal. Observe that $Z$ is $\Gamma$-invariant, and therefore, so is its complement $\widetilde{V}_{0}$ (and by a dual argument also $V_{0}$ ).

The following general result justifies that the angle between the spaces $V_{0}$ and $\widetilde{V}_{0}$ is always positive.

Lemma 4.3 (see $[17$, Lemma 3.3]). Let $Z, V, \widetilde{V}$ be closed subspaces of a Hilbert space $H$. If $H=V \oplus Z=\widetilde{V} \stackrel{\perp}{\oplus} Z$, then $R(\widetilde{V}, V)>0$.

We now have all the tools to prove our main result:

Proof of Theorem 1.5. It is clear that $(1) \Rightarrow(4) \Rightarrow(3)$. By Theorem 3.3 and (4.1) it follows easily that $(3) \Rightarrow(2)$. It remains to show that $(2) \Rightarrow(1)$.

Assume that (2) holds, and recall that $V_{0}$ and $\widetilde{V}_{0}$ are $\Gamma$-invariant. Hence, by (4.1) and (3.2) we have

$$
\begin{aligned}
D & =D_{\Psi, \widetilde{\Psi}}(\xi)=\operatorname{dim} \mathcal{F}(\xi)=\operatorname{dim} \widetilde{\mathcal{F}}(\xi)=\operatorname{dim} J_{V_{0}}(\xi) \\
& =\operatorname{dim} J_{\widetilde{V}_{0}}(\xi), \quad \text { a.e. } \xi \in \mathcal{D} .
\end{aligned}
$$

Now, Proposition 2.4 gives the existence of functions $\varphi^{1}, \ldots, \varphi^{D} \in V_{0}$ so that $\left\{\varphi_{\gamma}^{d}\right\}_{d, \gamma}$ is an ONB of $V_{0}$. Thus, $\left\{V_{J}\right\}_{J \in \mathbb{Z}}$ is an MRA, and likewise 
$\left\{\widetilde{V}_{J}\right\}_{J \in \mathbb{Z}}$. Furthermore, by (4.2) and Lemma 4.3 we must have $R\left(\widetilde{V}_{0}, V_{0}\right)>0$, and therefore we can apply Corollary 2.17 to conclude that $\left\{V_{J}, \widetilde{V}_{J}\right\}_{J \in \mathbb{Z}}$ are actually biorthogonal MRA's. This proves (1) and establishes the theorem.

Combining Theorems 3.3, 3.4 and 1.5 we obtain as well the following corollary.

COROLlary 4.4. If $\Psi=\left\{\psi^{1}, \ldots, \psi^{L}\right\}$ is a Riesz wavelet family associated with an $M R A$, then $L=D(|\operatorname{det} M|-1)$ for some $D \geq 1$. Moreover, there exists $\widetilde{\Psi}=\left\{\widetilde{\psi}^{1}, \ldots, \widetilde{\psi}^{L}\right\} \subset L^{2}\left(\mathbb{R}^{n}\right)$ such that $(\Psi, \widetilde{\Psi})$ is a pair of biorthogonal wavelet families arising from biorthogonal MRA's.

When $L=|\operatorname{det} M|-1$, Theorem 1.5 admits other equivalent formulations from similar arguments as in Theorem 3.5.

Corollary 4.5. Suppose $L=|\operatorname{det} M|-1$ and $\Psi, \widetilde{\Psi}$ are biorthogonal wavelet families in $L^{2}\left(\mathbb{R}^{n}\right)$. Then $(\Psi, \widetilde{\Psi})$ are associated with biorthogonal MRA's if and only if one of the following holds:

(1) $D_{\Psi, \widetilde{\Psi}}(\xi) \neq 0$, a.e. $\xi \in \mathcal{D}$.

(2) $\sum_{l=1}^{L} \sum_{j=1}^{\infty} \sum_{\gamma \in \Gamma}\left|\widehat{\psi}^{l}\left(M^{* j}(\xi+\gamma)\right)\right|^{2}>0$, a.e. $\xi \in \mathcal{D}$.

(3) $\sum_{l=1}^{L} \sum_{j=1}^{\infty} \sum_{\gamma \in \Gamma}\left|\widehat{\widetilde{\psi}}^{l}\left(M^{* j}(\xi+\gamma)\right)\right|^{2}>0$, a.e. $\xi \in \mathcal{D}$.

5. Further remarks and examples. In this last section we complete the results in the paper with various comments and examples illustrating the sharpness of our theorems.

1. For any positive integer $L \geq 1$, there exist orthonormal wavelet families with exactly $L$ elements: $\Psi=\left\{\psi^{1}, \ldots, \psi^{L}\right\}$ (see [11]). Thus, the condition $L=D(|\operatorname{det} M|-1)$ may not hold in some situations, in which case we necessarily have non-MRA wavelets.

2. There exist orthonormal wavelet families $\Psi=\left\{\psi^{1}, \ldots, \psi^{|\operatorname{det} M|-1}\right\}$ so that condition (3.6) in Theorem 3.5 does not hold (while the space $V_{0}=$ $\overline{\operatorname{span}}\left\{\psi_{j, \gamma}^{l}\right\}_{j<0, \gamma, l}=\overline{\operatorname{span}}\left\{\psi_{j, \gamma}\right\}_{j \geq 0, \gamma, l}^{\perp}$ is trivially shift-invariant). In the 1dimensional dyadic case the typical example is the Journé wavelet [16, p. 64], [17]. Similar examples in higher dimensions, for matrices $M$ with $|\operatorname{det} M|$ $=2$, were presented in $[8, \S 5]$.

3. Conversely, there exist Riesz wavelets $\psi \in L^{2}(\mathbb{R})$ satisfying (3.6) and for which $V_{0}$ cannot be shift-invariant [17]. Such examples are due to Zalik [22] and consist of (compactly supported) deformations of the Haar wavelet which "cannot be obtained by an MRA". We observe that the concepts of a wavelet "obtained by an MRA" [22] and "arising from an MRA" [16] are actually equivalent, as was shown by the first author in [5].

4. Theorem 3.5 cannot hold if we let $L=D(|\operatorname{det} M|-1)$ and $D>1$. In fact, for $|\operatorname{det} M|=2$ there exist orthonormal wavelet families $\Psi=\left\{\psi^{1}, \ldots, \psi^{2^{L}}\right\}$, 
with arbitrary $L \geq 1$, so that (3.6) holds (and $V_{0}$ is shift-invariant), but not arising from an MRA. Such constructions were presented in [8, Example 5.5], and were of the form $\Psi=\left\{\chi_{T_{1}}^{\vee}, \ldots, \chi_{T_{2} L}^{\vee}\right\}, L \geq 1$, where the sets $\left\{T_{l}\right\}_{l=1}^{2^{L}}$ form a suitable partition of $M^{* L} T$, and $T$ is a fixed wavelet set in $\mathbb{R}^{n}$. For these examples we showed that

$$
D_{\Psi}(\xi) \geq 2^{L}-1>0, \quad \text { a.e. } \xi \in \mathcal{D},
$$

while $\Psi$ arises from an MRA (of multiplicity $2^{L}$ ) iff $\psi=\chi_{T}^{\vee}$ arises from an MRA (see [8, Proposition 5.8]).

5. The dimension function associated with orthonormal wavelets has been studied by various authors in different contexts. In [6] there is a large collection of examples, in one and higher dimensions, of dimension functions taking arbitrarily large values, or even being unbounded. We point out that when $\psi$ is a Riesz wavelet which is not orthonormal, then the "dimension function" $D_{\psi}(\xi)$ is no longer integer-valued (see, e.g., Example 2.18 above).

6 . Concerning the results in $\S 4$, any pair of biorthogonal wavelets with very mild smoothness and decay properties will satisfy the conditions of Theorem 1.5. This result has been shown by Auscher [2, Theorem 10.1], see also [8, Corollary 4.16].

7. There also exist examples of biorthogonal wavelets (not necessarily orthonormal) which do not arise from biorthogonal MRA's. Such examples do not seem to appear in the literature, so for completeness we give a simple construction in the 1-dimensional dyadic situation. The construction follows an idea of X. Wang in the orthonormal setting. We refer to [21, Example 3.65$, p. 79$]$ for the elementary verification of the properties stated.

Let $0 \leq b(\xi) \leq 1, \xi \in[6 \pi / 7,8 \pi / 7)$, be an arbitrary measurable function, and let $0<\alpha<1$. We define $\widehat{\psi}(\xi)=e^{i \xi / 2}|\widehat{\psi}(\xi)|$, where

$$
|\widehat{\psi}(\xi)|= \begin{cases}1, & 4 \pi / 7 \leq|\xi|<6 \pi / 7, \\ b(\xi)^{\alpha}, & 6 \pi / 7 \leq \xi<8 \pi / 7, \\ (1-b(\xi / 4))^{\alpha}, & 24 \pi / 7 \leq \xi<32 \pi / 7, \\ (1-b(\xi+2 \pi))^{1-\alpha}, & -8 \pi / 7 \leq \xi<-6 \pi / 7, \\ b(\xi / 4+2 \pi)^{1-\alpha}, & -32 \pi / 7 \leq \xi<-24 \pi / 7 .\end{cases}
$$

We define $\widehat{\widetilde{\psi}}(\xi)=e^{i \xi / 2}|\widehat{\widetilde{\psi}}(\xi)|$ in a completely analogous way, except that we replace $\alpha$ by $\beta=1-\alpha$. Since (2.19) holds for $\varrho=\psi, \widetilde{\psi}$, the systems 
$\left(\psi_{j, k}\right)_{j, k \in \mathbb{Z}},\left(\widetilde{\psi}_{j, k}\right)_{j, k \in \mathbb{Z}}$ are Bessel. By similar computations to those in [21],

$$
\begin{aligned}
& \sum_{j \in \mathbb{Z}} \widehat{\psi}\left(2^{j} \xi\right) \overline{\widehat{\widetilde{\psi}}\left(2^{j} \xi\right)}=1, \quad \text { a.e. } \xi \in \mathbb{R}, \\
& t_{q}(\xi)=\sum_{j \geq 0} \widehat{\psi}\left(2^{j} \xi\right) \overline{\widehat{\widetilde{\psi}}\left(2^{j}(\xi+2 \pi q)\right)}=0, \quad \text { a.e. } \xi \in \mathbb{R}, q \in 2 \mathbb{Z}+1,
\end{aligned}
$$

hence by the results of [13], $\left(\psi_{j, k}\right)_{j, k \in \mathbb{Z}},\left(\widetilde{\psi}_{j, k}\right)_{j, k \in \mathbb{Z}}$ is a pair of dual frames. Furthermore, by a direct calculation we also have

$$
\begin{aligned}
\sum_{k \in \mathbb{Z}} \widehat{\psi}(\xi+k) \overline{\widehat{\widetilde{\psi}}(\xi+k)} & =1, \quad \text { a.e. } \xi \in \mathbb{R}, \\
\sum_{k \in \mathbb{Z}} \widehat{\psi}\left(2^{j}(\xi+k)\right) \overline{\widehat{\widetilde{\psi}}(\xi+k)} & =\sum_{k \in \mathbb{Z}} \widehat{\psi}(\xi+k) \overline{\widehat{\widetilde{\psi}}\left(2^{j}(\xi+k)\right)} \\
& =0, \quad \text { a.e. } \xi \in \mathbb{R}, j \geq 1,
\end{aligned}
$$

which shows biorthogonality of $\left(\psi_{j, k}\right)_{j, k \in \mathbb{Z}},\left(\widetilde{\psi}_{j, k}\right)_{j, k \in \mathbb{Z}}$ (see [16, Ch. 3.1]). This shows that $(\psi, \widetilde{\psi})$ is a biorthogonal wavelet pair. Moreover, $D_{\psi, \widetilde{\psi}}(\xi)$ is the usual Journé wavelet dimension function, i.e.,

$$
D_{\psi, \widetilde{\psi}}(\xi)= \begin{cases}2, & |\xi|<2 \pi / 7, \\ 1, & 2 \pi / 7<|\xi|<4 \pi / 7 \text { or } 6 \pi / 7<|\xi|<\pi, \\ 0, & 4 \pi / 7<|\xi|<6 \pi / 7 .\end{cases}
$$

Thus, $(\psi, \widetilde{\psi})$ cannot arise from a pair of biorthogonal MRA's. A limiting case of this construction corresponds to $\alpha \rightarrow 0$ and $b \equiv 1$. Then one obtains the wavelet sets in Figure 5.1.
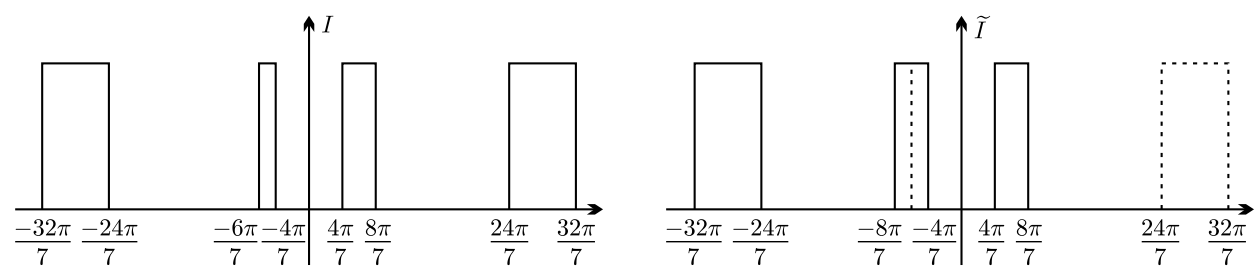

Fig. 5.1. The functions $|\widehat{\psi}|=\chi_{I}$ and $|\widehat{\widetilde{\psi}}|=\chi_{\widetilde{I}}$ when $\alpha=0$ and $b \equiv 1$

8. Finally, we would like to recall a fundamental open question in this subject: does any pair of biorthogonal MRA's have an associated pair of biorthogonal wavelets? In particular, is it true that the Cohen-Daubechies algorithm $[9,10]$ applied to any pair of biorthogonal MRA's always yields biorthogonal wavelets? As pointed out in Example 2.18, the biorthogonality follows automatically and the problem boils down to the verification of 
the Bessel condition (see also [12, p. 268]). It is known that the answer to the above question is affirmative under a relatively mild decay assumption on the scaling functions (see [10, Theorem 3.8]), where the assumption of compactly supported scaling functions can be easily relaxed. However, the general situation seems to remain open, with no counterexamples of wavelet systems obtained by the Cohen-Daubechies algorithm which do not satisfy the Bessel condition.

\section{References}

[1] A. Aldroubi, Oblique projections in atomic spaces, Proc. Amer. Math. Soc. 124 (1996), 2051-2060.

[2] P. Auscher, Solutions to two problems on wavelets, J. Geom. Anal. 5 (1995), 181-237.

[3] C. de Boor, R. A. DeVore and A. Ron, The structure of finitely generated shiftinvariant spaces in $L_{2}\left(\mathbb{R}^{d}\right)$, J. Funct. Anal. 119 (1994), 37-78.

[4] M. Bownik, The structure of shift-invariant subspaces of $L^{2}\left(\mathbb{R}^{n}\right)$, ibid. 177 (2000), 282-309.

[5] -, Riesz wavelets and generalized multiresolution analyses, Appl. Comput. Harmon. Anal. 14 (2003), 181-194.

[6] M. Bownik, Z. Rzeszotnik and D. Speegle, A characterization of dimension functions of wavelets, ibid. 10 (2001), 71-92.

[7] L. Brandolini, G. Garrigós, Z. Rzeszotnik and G. Weiss, The behaviour at the origin of a class of band-limited wavelets, in: Contemp. Math. 247, Amer. Math. Soc., Providence, 1999, 75-91.

[8] A. Calogero and G. Garrigós, A characterization of wavelet families arising from biorthogonal MRA's of multiplicity d, J. Geom. Anal. 11 (2001), 187-217.

[9] A. Cohen and I. Daubechies, A stability criterion for biorthogonal wavelet bases and their related subband coding scheme, Duke Math. J. 68 (1992), 313-335.

[10] A. Cohen, I. Daubechies and J.-C. Feauveau, Biorthogonal bases of compactly supported wavelets, Comm. Pure Appl. Math. 45 (1992), 485-560.

[11] X. Dai, D. Larson and D. Speegle, Wavelet sets in $\mathbb{R}^{n}$, J. Fourier Anal. Appl. 3 (1997), 451-456.

[12] I. Daubechies, Ten Lectures on Wavelets, SIAM, Philadelphia, 1992.

[13] M. Frazier, G. Garrigós, K. Wang and G. Weiss, A characterization of functions that generate wavelet and related expansion, J. Fourier Anal. Appl. 3 (1997), 883-906.

[14] G. Gripenberg, A necessary and sufficient condition for the existence of a father wavelet, Studia Math. 114 (1995), 207-226.

[15] H. Helson, Lectures on Invariant Subspaces, Academic Press, New York, 1964.

[16] E. Hernández and G. Weiss, A First Course on Wavelets, CRC Press, Boca Raton, 1996.

[17] H.-O. Kim, R.-Y. Kim and J.-K. Lim, Characterizations of biorthogonal wavelets which are associated with biorthogonal multiresolution analyses, Appl. Comput. Harmon. Anal. 11 (2001), 263-272.

[18] D. Larson, W.-S. Tang and E. Weber, Riesz wavelets and multiresolution structures, in: Proc. of the SPIE 2001, Vol. 4478, 254-262.

[19] Z. Rzeszotnik, Characterization theorems in the theory of wavelets, Ph.D. thesis, Washington Univ., 2000. 
[20] M. Unser and A. Aldroubi, A general sampling theory for nonideal acquisition devices, IEEE Trans. Signal Process. 46 (1998), 1130-1133.

[21] X. Wang, The study of wavelets from the properties of their Fourier transforms, Ph.D. thesis, Washington Univ., 1995.

[22] R. A. Zalik, Riesz bases and multiresolution analyses, Appl. Comp. Harmon. Anal. 7 (1999), 315-331.

Department of Mathematics

Departamento de Matemáticas C-XV

University of Michigan

Universidad Autónoma de Madrid

525 East University

Ciudad Universitaria Cantoblanco

Ann Arbor, MI 48109, U.S.A.

28049, Madrid, Spain

E-mail: marbow@math.lsa.umich.edu

E-mail: gustavo.garrigos@uam.es

Received March 28, 2002 